\title{
Phages fight alcoholic hepatitis
}

Alcoholic hepatitis is a lifethreatening disease, for which current corticosteroid therapy is only mildly effective. Now, writing in Nature, Schnabl and colleagues demonstrate that bacteriophages may be harnessed to attenuate ethanol-induced liver disease in mice, through modulation of the gut microbiome.

Previous studies in mice have shown that alcohol-related liver disease can be transmitted via faecal microbiota. However, little is known about the microorganisms and microbial factors that mediate this process.

To understand this, Schnabl and colleagues first analysed differences in the faecal microbiota composition of patients with alcoholic hepatitis or alcohol use disorder compared with controls. 16S rRNA sequencing of faecal microbiota revealed a substantial increase in the proportion of Enterococcus species in patients with alcoholic hepatitis, compared with those with alcohol use disorder or non-alcoholic control subjects. Additional quantitative PCR analysis indicated an approximate 2,700-fold increase in Enterococcus faecalis in alcoholic hepatitis faecal samples compared with controls.

E. faecalis secretes a bacterial exotoxin, named cytolysin, that exhibits lytic activity against
Gram-positive bacteria and also eukaryotic cells. The presence phage gavage of cytolysin-positive (cytolytic) one day

prior to an ethanol binge decreased liver cytolysin levels and abolished ethanol-induced liver injury and steatosis

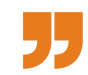
compared with only $3.8 \%$ of cytolysin-negative patients.

Next, to determine whether the authors gavaged mice with

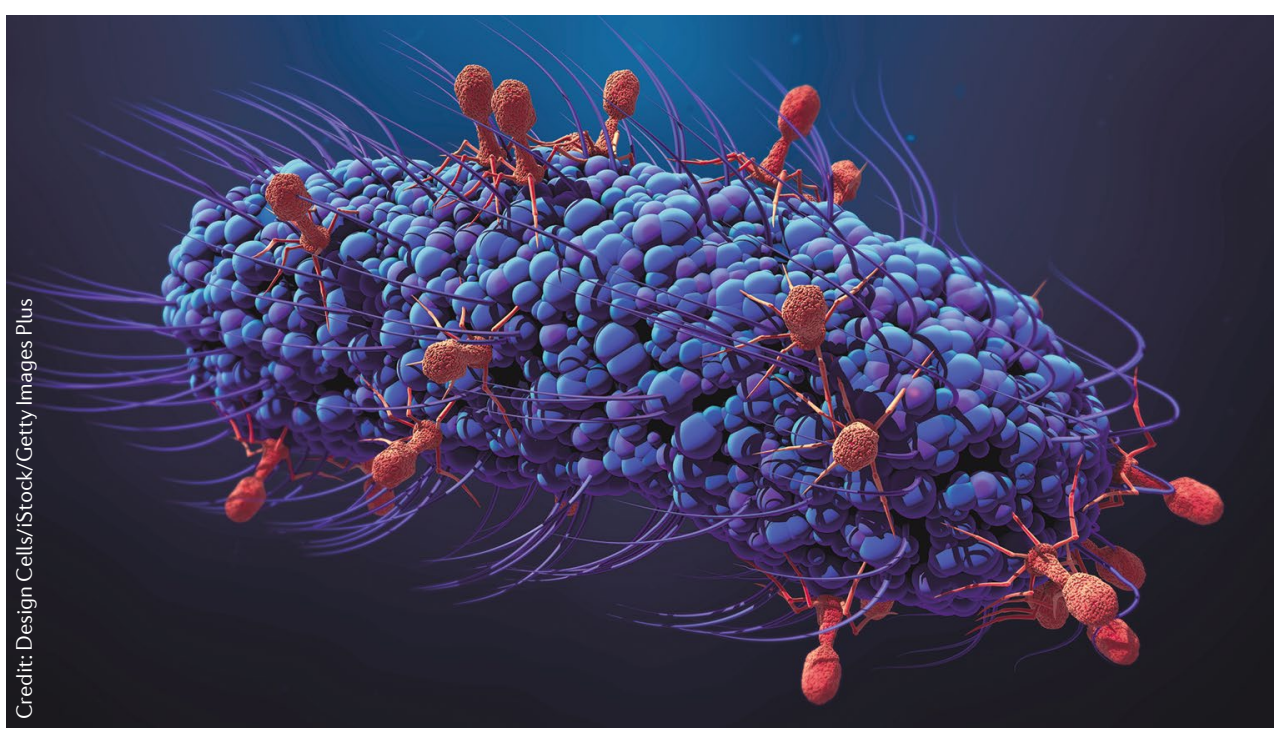

E. faecalis correlated with the severity of liver disease and with mortality in patients with alcoholic hepatitis: $89 \%$ of cytolysin-positive patients with alcoholic hepatitis died within 180 days of hospital admission, cytolysin contributes to liver damage, either a cytolytic or non-cytolytic E. faecalis strain and placed them on a chronic-binge ethanol diet. Those mice receiving cytolytic E. faecalis exhibited increased levels of cytolysin in the liver and developed more severe ethanolinduced liver injury, increased hepatic steatosis and increased liver inflammation compared with mice receiving a non-cytolytic strain.

Consistent with these findings, gnotobiotic mice transplanted with faeces from cytolysin-positive patients experienced more severe ethanol-induced liver injury, steatosis, inflammation and fibrosis, than mice given faeces from cytolysin-negative patients.

Mechanistic studies indicated that ethanol-induced changes in the gut barrier likely enable translocation of cytolytic E. faecalis from the intestine to the liver, resulting in increased levels of liver cytolysin. In isolated mouse hepatocytes, cytolysin induced cell death.

Next, the authors focused their studies on a strain of mouse (Atp $4 a^{S l / s l}$ mice), which exhibits an overgrowth of intestinal enterococci, associated with increased susceptibility to alcoholic hepatitis. Four phages capable of lysing a cytolytic E. faecalis strain found in the Atp $4 a^{\text {sl/sl }}$ mice were isolated from sewage water. Gavaging Atp $4 a^{\text {Sl/Sl }}$ mice with these phages resulted in less severe liver injury, steatosis and inflammation after chronic ethanol feeding, compared with mice gavaged with control phages or vehicle. Importantly, the phages significantly reduced levels of cytolysin in the liver as well as faecal amounts of Enterococcus, without affecting the overall composition of the faecal microbiome based on $16 \mathrm{~S}$ rRNA sequencing analysis.

Finally, the authors assessed the therapeutic potential of bacteriophages in the treatment of ethanol-induced liver disease. Cytolytic E. faecalis strains were cultured from faecal samples from patients with alcoholic hepatitis and then lytic phages against these bacterial strains were again isolated from sewage water. In gnotobiotic mice colonized with faeces from cytolysin-positive patients, phage gavage 1 day before an ethanol binge decreased liver cytolysin levels and abolished ethanol-induced liver injury and steatosis.

These findings provide hope of a novel effective therapy for the treatment of alcoholic hepatitis. The authors are currently expanding their biobank of phages against cytolytic isolates from patients with alcoholic hepatitis and have plans for a future phase I safety trial.

\section{Sarah Crunkhorn}

ORIGINAL ARTICLE Duan, Y. et al. Bacteriophage targeting of gut bacterium attenuates alcoholic liver disease. Nature https://doi.org/10.1038/ s41586-019-1742-x (2019) 